\title{
Servo Controller Design For An Optical Disk Drive Using Fuzzy Control Algorithm
}

\author{
Jia-Yush Yen§ Chuan-Shan Lin† Chung-Han Li†Yung-Yaw Chen \
}

\begin{abstract}
In this paper, the fuzzy control of an optical disk drive compound actuator is presented. Fuzzy control logic is especially suited for the control of compound actuators. Many problems associated with the compound actuator control are addressed. The coarse actuator generally has very different resolution than the fine, and the fine actuator is limited by its stroke. All these problems are not treated in the traditional linear or nonlinear control theories. The fuzzy control logic, on the other hand, provides an easy way for implementing these specifications. Results for fuzzy controller applications with different configurations are compared. A hybrid control algorithm is then proposed. It is shown that the common approach of using two stage control for track accessing and track following can be combined using fuzzy control algorithm.
\end{abstract}

\section{Introduction}

In this paper, a fuzzy control algorithm for the compound actuator is presented. The "compound actuator", sometimes called the "dual actuator" or the "piggy actuator", is widely used in the optical disk drive servo system [1]. Data in the optical disk drive is recorded on the disk surface as small areas of different reflectivities alone concentric or spiral tracks. Common optical disks records $325 \mathrm{Kbits} / \mathrm{mm}^{2}$. The data tracks are $2 \mu \mathrm{m}$ apart [2]. With a 5 and $1 / 4$ in disk, the actuator has to move the head across a $5 \mathrm{~cm}$ stroke and position it within $0.1 \mu \mathrm{m}$ accuracy. Conventional actuators do not achieve both the large stroke and the fine resolution. A compound actuator is thus necessary.

The compound actuator is consists of a fine actuator mounted on the back of a coarse actuator. The fine actuator has a fast response and high resolution, while the coarse actuator can access very large movements. The control of the compound actuator, however, represents certain difficulties. First of all, both actuators are trying to follow the same tracking signal, which means it is not clear for the controller how to devide the effort between the actuators. A general approach is to make the fine actuator following the actual tracking error, and make the coarse actuator following the displacement between the two actuators [3]. Because the coarse actuator is heavier, it will automatically follow the slower motions and leave the fast movement to the fine. Tunning the controller is almost entirely by experience. Some companies tried to use high-pass and low-pass filters to separate the signals [4]. A more advanced approach is to use the frequency shaped LQG/LTR technique [5]. The controller design is based

$\S$ Associate Professor, Department of Mechanical Engineering, National Taiwan University

$\dagger$ Graduate Student, Department of Mechanical Engineering, National Taiwan University

I Associate Professor, Department of Electrical Engineering, National Taiwan University 
upon a frequency domain criterion. By carefully choosing the weighting function, one can effectively shape the overall system responses into desired shape. This approach uses the basic linear system assumption that all the signals are measured with infinitely fine resolution. The case in the optical disk drive, however, is different. The tracking error signal for the fine actuator feedback is generated by the preformatted servo coding on the disk surface. The displacement between the two actuators, on the other hand, is measured either by a photo-encoder or a capacitance sensor. There is obviously a big difference between the resolutions of the two. These problems are not addressed by the conventional control theories; however, they can easily be treated with fuzzy control algorithms. The frequency domain criteria can be translated into different emphases on the error and the change of error. The different resolutions can be translated into membership functions with different sizes. The restrictions for the limited fine actuator stroke can be incorporated as an additional rule in the rule base.

Two implementation methods are compared in the simulation. Observing the advantages of the two approaches, a hybrid control algorithm is then proposed. The usual approach of using a two stage track accessing and a separate track following controller [6] is not necessary. The hybrid fuzzy control algorithm posseses the advantages of both algorithms.

\section{System Modeling and Identification}

In this section, system modeling and identification will be described. Assuming the response of the motor driver is fast enough to be treated as a pure gain, the compound actuator can be represented as in Fig.1 [5].

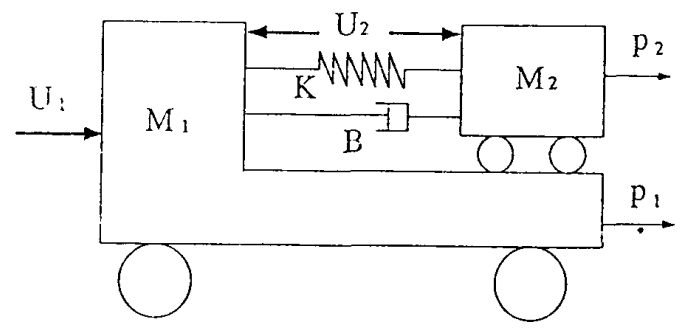

Fig.1 Compound actuator model

$p_{1}$ is the position of the coarse actuator, and $p_{2}$ is the position of the fine actuator. $K$ and $B$ are the coupling spring constant and damping ratio, respectively. The state space representation of the system is expressed as

$$
\dot{x}=\mathbf{A} x+\mathbf{B} u, \quad \mathbf{y}=\mathbf{C} x+\mathbf{D} u,
$$


where $x=\left[\begin{array}{llll}x_{1} & x_{2} & x_{3} & x_{4}\end{array}\right]^{\prime}, u=\left[\begin{array}{ll}u_{1} & u_{2}\end{array}\right]^{\prime}$,

$$
\begin{gathered}
\mathbf{A}=\left[\begin{array}{cccc}
0 & 1 & 0 & 0 \\
-\frac{K}{M_{1}} & -\frac{B}{M_{1}} & \frac{K}{M_{1}} & \frac{B}{M_{1}} \\
0 & 0 & 0 & 1 \\
\frac{K}{M_{2}} & \frac{B}{M_{3}} & -\frac{K}{M_{2}} & -\frac{B}{M_{2}}
\end{array}\right], \mathbf{B}=\left[\begin{array}{cc}
0 & 0 \\
\frac{1}{M_{1}} & -\frac{1}{M_{1}} \\
0 & 0 \\
0 & \frac{1}{M_{2}}
\end{array}\right], \\
\text { and } \mathbf{C}=\left[\begin{array}{cccc}
0 & 0 & 1 & 0 \\
-1 & 0 & 1 & 0
\end{array}\right], \mathbf{D}=0 .
\end{gathered}
$$

$x_{1}$ in (1) represents the position of the coarse actuator, and $x_{3}$ represents the position of the fine actuator. $u_{1}$ is the input to the coarse actuator, and $u_{2}$ is the input to the fine actuator. Since only the fine position and the relative distance between the two actuators are measured, the output $y_{1}$ and $y_{2}$ are defined as $y_{1}=x_{3}$, and $y_{2}=x_{1}-x_{3}$.

The system transfer matrix is then derived as

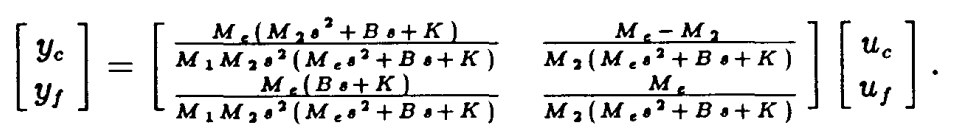

Notice in (3), $y_{c}$ is the coarse actuator output position. $y_{f}$ is the fine actuator output position. $u_{c}$ now represents the coarse actuator input. $u_{f}$ is the fine actuator input, and $M_{e}=\frac{M_{1} M_{3}}{M_{1}+M_{2}}$ is the equivalent mass for the coupling resonance.

The coarse actuator in our experimental drive uses a worm gear output. The equivalent mass $M_{1}$ for the coarse actuator is therefore very much larger than $M_{2}$, and one can say $M_{e} \rightarrow M_{2}$. Equation (3) can thus be simplified as

$$
\left[\begin{array}{l}
y_{c} \\
y_{f}
\end{array}\right]=\left[\begin{array}{cc}
\frac{1}{M_{1} o^{2}} & 0 \\
\frac{(B \cdot+K)}{M_{1} o^{2}\left(M_{2} o^{2}+B o+K\right)} & \frac{1}{\left(M_{2} o^{2}+B o+K\right)}
\end{array}\right]\left[\begin{array}{l}
u_{c} \\
u_{f}
\end{array}\right] .
$$

Parameter identification is performed experimentally. The fine actuator position is measured with an Keyence PA-1800 laser displacement sensor with $5 \mu m$ resolution. The coarse actuator position is measured by a Keyence LB-70 displacement sensor with $40 \mu \mathrm{m}$ resolution. By linear system assumption, the four elements of the transfer function matrix are separately identified. The one-one element is easily measured by looking at the frequency response of the coarse actuator due to the coarse excitation. The effect of fine excitation to the coarse is neglected. The two-two element is then measured by fixing the coarse actuator and examining the fine response to the fine input. A HP3563A control system analyzer is used and the responses as well as their curve fits are plotted in Fig.2a-2b. Since the two-one element contains no new parameter, all the elements in (4) are obtained.

Since there is a significant back lash exists in the worm gear, the frequency response curve for the coarse actuator only covers relatively low frequency area. 


\section{Generation of Fuzzy Control Rules}

The controller design is based on the fuzzy algorithm proposed by Zadeh [7]. Both the error signal $e(k)$ and the change of error signal $e_{c}(k)$ are used for controller inputs. Scaling factors are introduced between the measurement space and the universe of discourse to aid in the tuning precess [8]. The structure of the compound actuator controller is shown in Fig.3

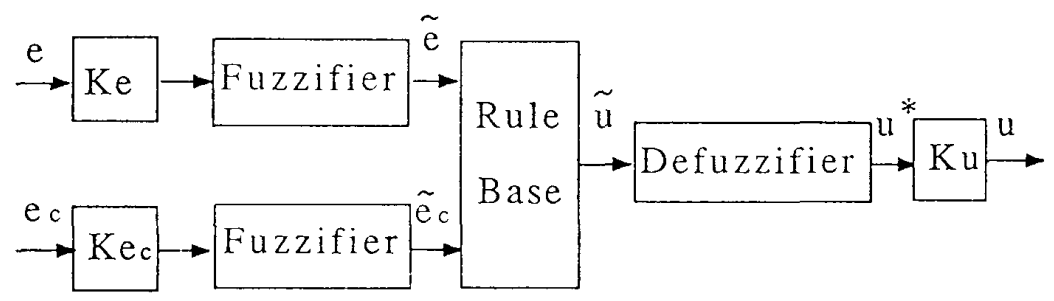

Fig.3 Fuzzy controller for compound actuator

where $K_{e}$ and $K_{e} c$ are the scaling factors for the error signal and the change of error signals, respectively. The fuzzy variables are chosen as shown in table I.

\section{Development of the Fuzzy Control Rule Base}

As mentioned in our introduction, the track following controller has to track large signals with very fine resolution. The fine actuator has to track small and fast motions, while the coarse actuator has to follow the larger movements. The fine actuator has a limited stroke, therefore the coarse actuator has to compensate for the insufficient movement. Because the fine actuator has a faster response, the controller should use the fine actuator whenever it is able to cover the movement. Although it is slower, the coarse actuator should also try to follow the reference so that the steady state displacement between the two actuators will approach zero. This criterion is based on two main reasons: 1 . The fine actuator should always have tolerances on both side during following so that signals in both directions can be followed. 2 . The power consumption of the fine actuator is proportional to the square of averaged displacement it is keeping. By keeping an zero average displacement, the power consumption is minimized.

The coarse actuator is modeled as a pure inertia system. Five quantization levels are used. The decision table is shown in table II. Some modifications are made to the fine controller to compensate for the spring and damper effect. The fine actuator transfer function is represented by

$$
\mathbf{Y}_{f}(s)=\frac{K_{f}}{M_{2} s^{2}+B s+K} U_{f}(s),
$$


where $K_{f}$ is a constant gain from the fine actuator input to the fine actuator position. Rewritting equation (5) as

$$
\ddot{y}(t)=\frac{1}{M_{2}}\left[-B \dot{y}(t)-K y(t)+K_{f} u(t)\right],
$$

and define $\hat{u}(t)=\frac{1}{M_{2}}\left[-B \dot{y}(t)-K y(t)+K_{f} u(t)\right]$, the fine actuator response becomes

$$
\ddot{y}(t)=\hat{u}(t) .
$$

Using $\hat{u}$ for intput, the fine actuator can also be treated as a pure inertia system, and Table II can again be used.

The following rules are then imposed to achieve the compound actuator control.

1). If the fine actuator is capable of handling the movement, then use fine actuator.

2). If the range exceeds the fine actuator stroke, then maintain the fine actuator at the stretched position and use the coarse actuator.

In the second rule, the fine actuator is kept at the stretched position to minimize the time required for the travel. This criterion is also necessary so that no excessive energy is wasted in pushing the fine actuator while it has already reached the end of its stroke.

\section{Simulation Results}

In order to illustrate the working principle for the compound actuator, two ways of feedback are compared.

In the first approach, the positions of both actuators are assumed to be directly measurable. The controller for the actuators can then use these position informations for feedback. This is comparable to the usual assumption. Fig.4a shows the step response. The coarse actuator has a rather slow dynamic response as shown in Fig.4b; and it takes about $2.3 \mathrm{sec}$ for the actuator to reach the desired position. Notice the sudden rising in the response in Fig.4a when the signal is close to the set point. This is the place when the response comes within the range of the fine actuator. The fine actuator will suddenly take off so that the overall response will reach the set point in a very short time. Fig.4c and $4 \mathrm{~d}$ shows the response to a filtered random signal. A large overshoot is observed for the coarse actuator when the reference signal changes direction.

In the second approach, the two actuator positions are no longer available. Instead, the tracking error for fine actuator and the displacement between the fine and coarse actuators are used. This is a more realistic assumption since only the off track error and the displacement between the two actuators are available in the disk drive application. Step response for this appraoch is shown in Fig.5a. Since the coarse actuator now does not have access to the actual offset, there is a very large overshoot. The response takes until $3.5 \mathrm{sec}$ to reach the desired position. The settling time for the coarse actuator 
(Fig.5b) is also very long. The case of random signal following (Fig.5c), however, showed less overshoot. We believe this is the result of a more conservative control due to the fact that the coarse controller can only be driven by the limited displacement between the two actuators. The driving force for the coarse is therefore limited. Since fine actuator will compensate for the remaining error anyway, the second approach is more effective. Also, in this case a stroke less than $\pm 0.8 \mathrm{~mm}$ stroke is necessary for the fine actuator (Fig.5d).

\section{Hybrid Control Algorithm}

The above results suggest that a hybrid algorithm would still provide a better control. The hybrid controller tries to use first algorithm for large step access and switches into the second algorithm for small signal following. The advantages of both of the previous algorithms can thus be combined. In order to obtain coarse position, an observer is used. Two rules are added to the rule base for the hybrid control:

1). If the error is very large, then use the estimated coarse position for feedback.

2). If the error is not very large, then use the displacement between the two actuators for coarse feedback.

3). If the fine actuator has reached the end of its stroke, then reduce the fine control effort to the amount just enough to keep it in position.

The third rule is added to minimize power consumption. The simulation results are shown in Fig.6a-d. The coarse actuator shows little overshoot in the step response, and the settling time is reduced to $2.8 \mathrm{sec}$.

\section{Conclusions}

In this paper, the application of fuzzy logic on the control of compound actuator is presented. With some modification to the existing fuzzy controller, very nice performancer can be achieved. It is shown that most of the basic design delimma associated with the compound actuator servo problem can be easily treated in the rule base for the fuzzy controller. Two implementation techniques were compared. It was shown that when the actuator outputs are avialable, the large step response has a very fast settling property. Large overshoots can occur in the random signal following. A more realistic approach is to use the tracking error for fine actuator feedback and the relative displacement for the coarse actuator feedback, and the simulation results showed a very large overshoot when large step reference signal was introduced. The random signal following responses, however, showed very satisfactory results. A hybrid control algorithm was then proposed to incorporate the first approach for large step movement and switches into the second approach when the setpoint is within the fine actuator stroke. The resultant control showed the both satisfactory performances for large step access and small signal following. 


\section{References}

1. Meyer,R.A., 1986, "Control system considerations in read/write optical disk drives", fifteenth annual symposium on incrememtal motion control systems and devices, June, pp.85-90.

2. Lloyd,P., and Fujitani,L., 1983, "New optical disk drive loads 1G byte on a 12-inch platter", MINI-MICRO systems, Oct., pp.277-282.

3. Imaino,W., and Bhushan,B., 1991, "Actuation Mechanisms in optical storage", Advances in information storage systems, vol.1, pp.375-404.

4. Kitani,H., and Tsunoda,Y., 1984, "Large-capacity optical disk files", Hitachi review, Vol.33, No.3, pp.109-114.

5. Yen.J.Y., Hallamasek,K., and Horowitz,R., 1990, "Track-following controller design for a compound disk drive actuator", ASME Trans. J. of Dynamic Systems, Measurement, and Control, Vol.112, Sep., pp.391-402.

6. Kaneko,R., 1987, "Magnetic and optical disk storage technology", JSME International Journal, Vol.30, No.260, Feb., pp.215-220.

7. Zadeh,L.A., 1968, "Fuzzy algorithm", Inform. and Control, Vol.12, pp.94-102.

8. Tang,K.L., and Mulholland,R.J., 1987, "Comparing fuzzy logic with classical controller design", IEEE Trans. on Systems, Man, and Cybernetics, Vol.SMC-17, No.6, Nov., pp.1085-1087.

9. Li,Y.F., and Lau,C.C., 1989, "Development of fuzzy algorithms for servo systems", IEEE Control System Magazine, April, pp.65-72. 
Table I.

\begin{tabular}{|l}
\hline The fuzzy mapping \\
\hline$\mu_{P B}(x)=\left\{\begin{array}{ll|}1, & \text { if } x \geq 1 ; \\
2(x-0.5), & \text { if } 0.5 \leq x \leq 1 ; \\
0, & \text { if } x \leq 0.5 .\end{array}\right.$ \\
$\mu_{P B}(x)= \begin{cases}0, & \text { if } x \geq 1 ; \\
1-2|x-0.5|, & \text { if } 0 \leq x \leq 1 ; \\
0, & \text { if } x \leq 0 .\end{cases}$ \\
\hline$\mu_{P B}(x)= \begin{cases}0, & \text { if } x \geq 0.5 ; \\
1-2|x|, & \text { if }-0.5 \leq x \leq 0.5 ; \\
0, & \text { if } x \leq-0.5 .\end{cases}$ \\
\hline$\mu_{P B}(x)= \begin{cases}0, & \text { if } x \geq 0 ; \\
1-2|x+0.5|, & \text { if }-1 \leq x \leq 0 ; \\
0, & \text { if } x \leq-1 .\end{cases}$ \\
\hline$\mu_{P B}(x)= \begin{cases}1, & \text { if } x-0.5 ; \\
2|x+0.5|, & \text { if }-1 \leq x \leq-0.5 ; \\
1, & \end{cases}$ \\
\hline
\end{tabular}

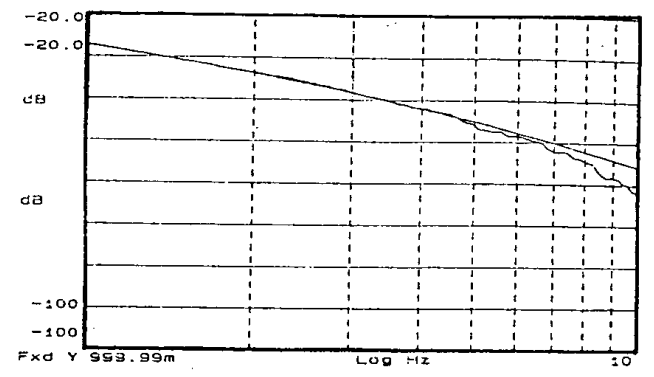

Fig.2a The frequency response of the coarse actuator

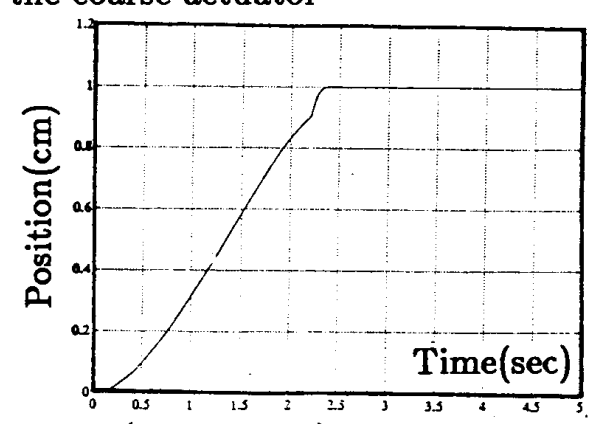

Fig.4a (Approach I) Fine actuator step response6

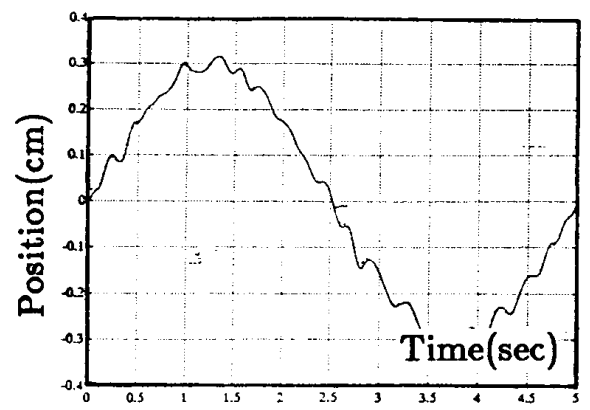

Fig.4c Random signal tracking response

\begin{tabular}{|c|c|c|c|c|c|}
\hline \multicolumn{6}{|c|}{ The decision table } \\
\hline$e, u, e_{c}$ & NB & NM & ZR & $\mathrm{PM}$ & $\overline{\mathrm{PB}}$ \\
\hline $\mathrm{PB}$ & 0 & $+\mathrm{S}$ & $+\mathrm{M}$ & $+\mathrm{L}$ & $+\mathrm{V}$ \\
\hline $\mathrm{PM}$ & $-S$ & 0 & $+\mathrm{S}$ & $+\mathrm{M}$ & $+\mathrm{L}$ \\
\hline $\mathrm{ZR}$ & $-M$ & $-S$ & 0 & $+\mathrm{S}$ & $+\mathrm{M}$ \\
\hline $\mathrm{NM}$ & $-\mathrm{L}$ & $-\mathrm{M}$ & $-\mathrm{S}$ & 0 & $-S$ \\
\hline $\mathrm{NB}$ & $-V$ & $-\mathrm{L}$ & $-M$ & $-S$ & 0 \\
\hline
\end{tabular}

Table II.

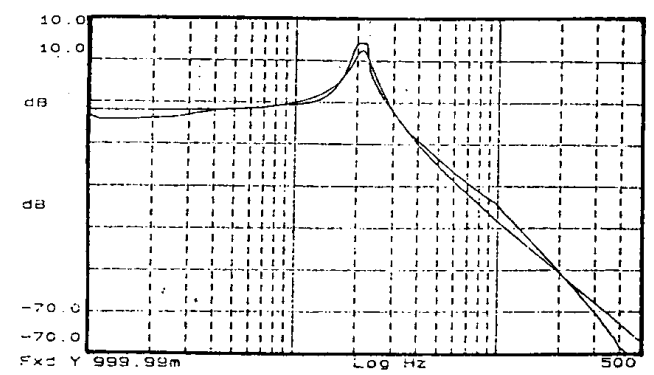

Fig.2b. The frequency response of the fine actuator

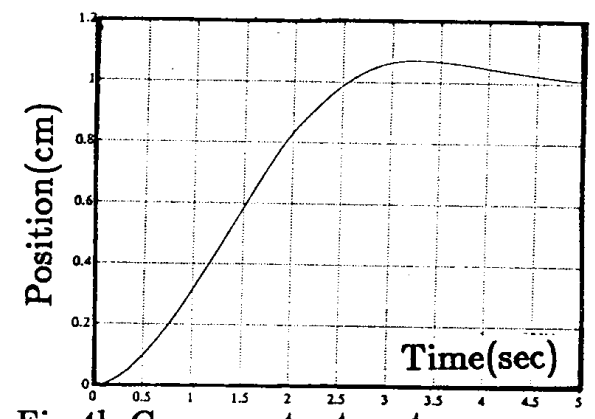

Fig.4b Coarse actuator step response

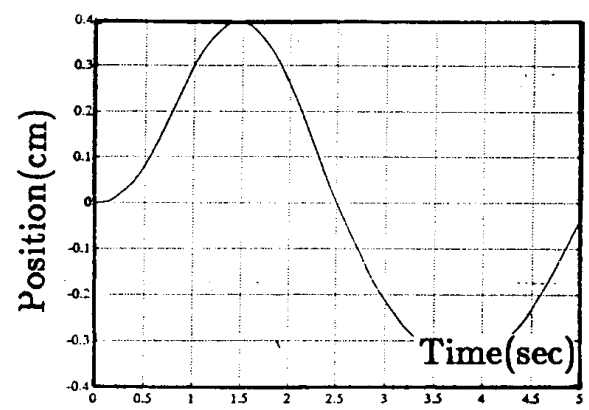

Fig.4d Coarse response to random signal tracking 


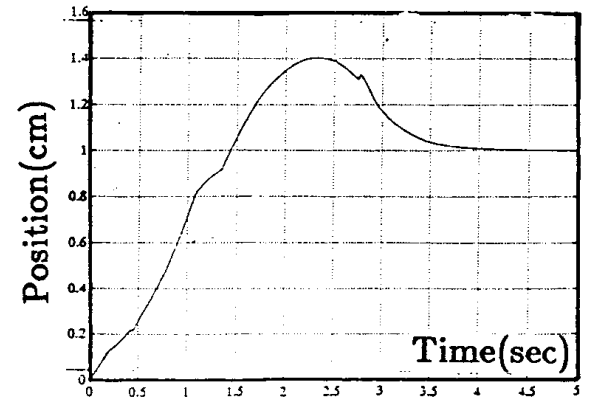

Fig.5a (Approach II) Fine actuator step response6

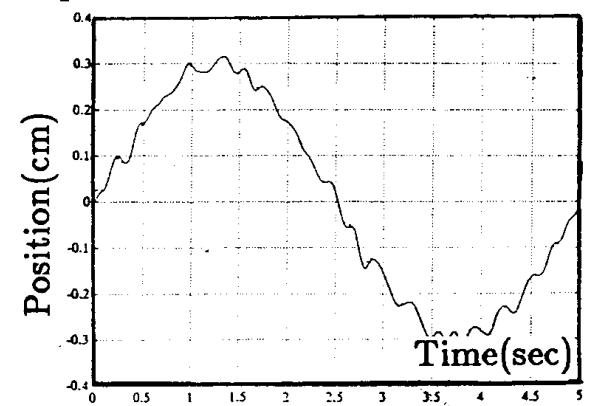

Fig.5c Random signal tracking response

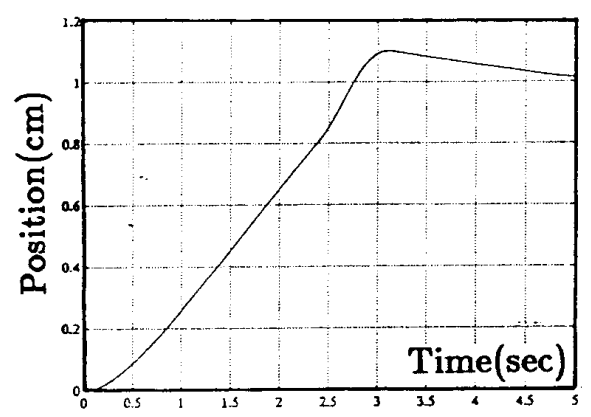

Fig.6a (Hybrid) Fine actuator step response6

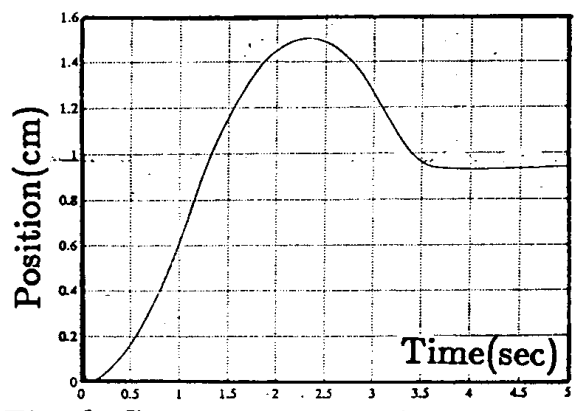

Fig.5b Coarse actuator step response

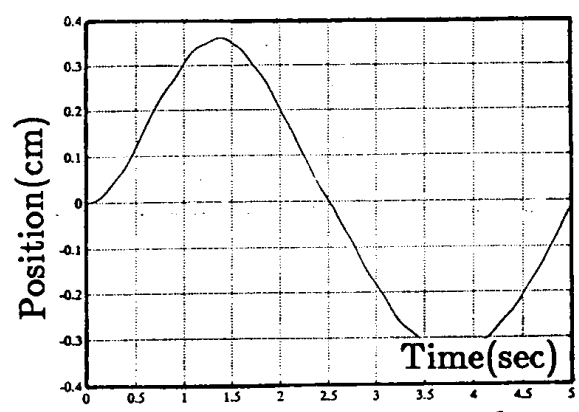

Fig.5d Coarse response to random signal tracking

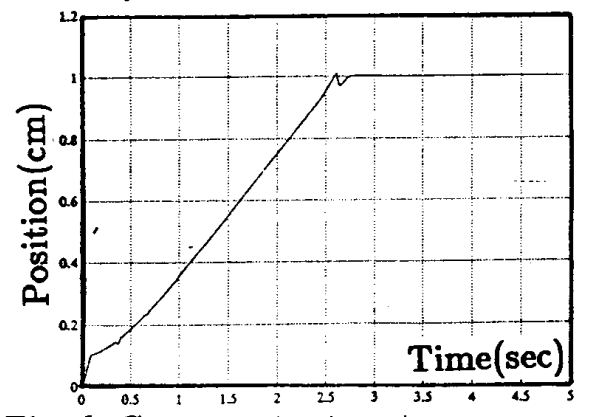

Fig.6b Coarse actuator step response 